\title{
On the Stabilization and Observer Design of Polytopic Perturbed Linear Fractional-Order Systems
}

\author{
Omar Naifar $\mathbb{D}^{1}$ and Abdellatif Ben Makhlouf $\mathbb{D}^{2,3}$ \\ ${ }^{1}$ Control and Energy Management Laboratory, National School of Engineering, Sfax University, Sfax, Tunisia \\ ${ }^{2}$ Department of Mathematics, College of Science, Jouf University, Sakaka, Aljouf, Saudi Arabia \\ ${ }^{3}$ Department of Mathematics, Faculty of Sciences of Sfax, Sfax University, Sfax, Tunisia \\ Correspondence should be addressed to Omar Naifar; email.naifar@gmail.com
}

Received 31 December 2020; Revised 3 March 2021; Accepted 5 March 2021; Published 24 March 2021

Academic Editor: Mehmet Emir Koksal

Copyright (c) 2021 Omar Naifar and Abdellatif Ben Makhlouf. This is an open access article distributed under the Creative Commons Attribution License, which permits unrestricted use, distribution, and reproduction in any medium, provided the original work is properly cited.

\begin{abstract}
In this paper, the problem of stabilization and observer design of parameter-dependent perturbed fractional-order systems is investigated. Sufficient conditions on the practical Mittag-Leffler and Mittag-Leffler stability are given based on the Lyapunov technique. Firstly, the problem of stabilization using the state feedback is developed. Secondly, under some sufficient hypotheses, an observer design which provides an estimation of the state is constructed. Finally, numerical examples are provided to validate the contributed results.
\end{abstract}

\section{Introduction}

Stability and control problems of nonlinear systems have drawn a great deal of attention from multiple fields of science and engineering (see $[1,2]$ and the references therein). On the other hand, fractional-order nonlinear systems (FONS) have become one of the most important subjects to be investigated on the control theory field. This includes fault estimation [1], observer design [3], stabilization [3, 4], stability [5, 6], finite-time stability (FTS) [7], neural networks [8], and stochastic systems [9].

Lyapunov stability of linear time-varying (LTV) systems and applications to control theory have received significant attention $[9,10]$. Parameter-dependent systems $[11,12]$ are acknowledged as one of important representations of these classes of systems. The use of Lyapunov functions is surely the main tool for solving the stability problem $[13,14]$. In order to get less conservative results, parameter-dependent Lyapunov functions have, in the past few years, been employed, and multiple techniques demand that these functions have been suggested for the stability and stabilization $[15,16]$. Furthermore, observer design has been a subject of interest, and a variety of methods has been developed for constructing nonlinear observers $[17,18]$. On the other hand, dealing with fractional-order systems, few works have been done to investigate parameter-dependent perturbed fractional-order systems (see [19]). Indeed, Abdellatif et al. in [19] have investigated the practical Mittag-Leffler stability (MLS) of nonlinear fractional-order systems depending on a parameter by using the Lyapunov techniques.

The study of linear systems with polytopic uncertainty has received a great deal of attention, and some significant results have been obtained (see [20-24]). In fact, authors in [20] have investigated stability of certain polytopic systems. With regard to fractional-order systems, authors in [21] have presented the problem of stability and stabilization for linear parameter-varying (LPV) polytopic systems with timevarying time delays. With regard to fractional-order systems, authors in [22] have investigated a new polytopic type uncertain state-space model for fractional-order linear systems.

In this work, the problem of stabilization and observer design of parameter-dependent perturbed fractional-order systems has been investigated. Up to now, the problem of stabilization and observer design of parameter-dependent 
perturbed integer-order systems has been tackled by several researchers. However, few works have been done to investigate such context on fractional-order systems. In this context, this paper presents a complete methodology to solve this problem. On the other hand, to the best of our knowledge, among all the existing works dealing with the parameter-dependent systems, no paper has treated the special class which is described in the present work. That is to say, the polytopic system means that the parameters are continuous functions with respect to time and their sum equal to 1 . On comparison, our polytopic system presents a perturbation term which is not the case in the work of [22]. Thus, we think that the contribution of our work is consistent.

In fact, the suggested polytopic fractional nonlinear system in this work is written as follows:

$$
\begin{aligned}
{ }_{C} D_{t_{0}, t}^{\alpha} w(t) & =A(\omega(t)) w(t)+B(\omega(t)) v(t)+f(t, \omega(t), w) \\
z & =C w(t),
\end{aligned}
$$

where ${ }_{C} D_{t_{0}, t}^{\alpha}$ is the Caputo fractional derivative operator of order $\alpha, w(t) \in \mathbb{R}^{n}$ is the state of the system, $v(t) \in \mathbb{R}^{p}$ is the control, $z(t) \in \mathbb{R}^{q}$ is the output, and the function $f: \mathbb{R}_{+} \times$ $\mathbb{R} \times \mathbb{R}^{n} \longrightarrow \mathbb{R}^{n}$ is the perturbation term. Matrices $A(\omega(t))$ and $B(\omega(t))$ belong to the convex envelope $\wp$ defined by

$$
\wp=\left\{\sum_{i=1}^{m} \omega_{i}(t) F_{i}, \omega_{i}(t) \in \mathbb{R}_{+} \text {and } \sum_{i=1}^{m} \omega_{i}(t)=1\right\},
$$

with $F_{i}$ are constant matrices and $C \in \mathbb{R}^{q \times n}$ is the output matrix.

The paper is organized as follows. Basic results related to the fractional calculus are presented in Section 2. After that, the main results, dealing with the problem of stabilization and observer design of parameter-dependent perturbed fractional-order systems, are shown in Section 3. Illustrative examples with simulation results are described in Section 4 to demonstrate the efficiency of the proposed scheme. Finally, conclusion is presented in Section 5.

\section{Problem Statement and Preliminaries}

In what follows, some general definitions and basic results regarding the fractional calculus have been shown [25].

Definition 1. Given an interval $[x, y]$ of $\mathbb{R}$, the RLFI of order $\alpha>0$ of a function $w \in L^{1}([x, y])$ is defined as

$$
I_{x}^{\alpha} w(u)=\frac{1}{\Gamma(\alpha)} \int_{x}^{u}(u-m)^{\alpha-1} w(\tau) \mathrm{d} m, \quad u \in[x, y],
$$

where $\Gamma(\alpha)=\int_{0}^{+\infty} e^{-m} m^{\alpha-1} \mathrm{~d} m$.

Definition 2. Given an interval $\left[d_{0}, y\right]$ of $\mathbb{R}$, the Caputo fractional derivative of order $\alpha$ of $p(t)$ is defined by

$$
{ }_{C} D_{d_{0}, u}^{\alpha} w(u)=I_{d_{0}}^{k-\alpha} w^{(k)}(u), \quad u \in\left[d_{0}, y\right],
$$

where $0<k-1<\alpha \leq k$.
When $0<\alpha<1$, the Caputo fractional derivative of order $\alpha$ of an absolutely continuous function $w$ on $\left[d_{0}, y\right]$ reduces to ${ }_{C} D_{d_{0}, u}^{\alpha} w(u)=\frac{1}{\Gamma(1-\alpha)} \int_{t_{0}}^{u}(t-m)^{-\alpha} w^{\prime}(m) \mathrm{d} m, \quad t \in\left[d_{0}, y\right]$.

By the next definition, a frequently used function in the resolution of fractional-order systems is presented. This function can be regarded as a generalization of the exponential function.

Lemma 1 (see [26]). Let $M \in \mathbb{R}^{p \times p}$ be a symmetric, positive definite matrix and $\alpha \in] 0,1[$. Then, the following relationship holds:

$$
\frac{1}{2}{ }_{C} D_{d_{0}, u}^{\alpha}\left(w^{T}(u) M w(u)\right) \leq w^{T}(u) M_{C} D_{d_{0}, u}^{\alpha} w(u) .
$$

Definition 3. The Mittag-Leffler function with two parameters is defined as follows:

$$
E_{m, v}(p)=\sum_{s=0}^{+\infty} \frac{p^{s}}{\Gamma(k m+v)}
$$

where $m>0, v>0$, and $p \in \mathbb{C}$. When $v=1$, one has $E_{m}(p)=E_{m, 1}(p)$; furthermore, $E_{1,1}(p)=e^{p}$.

Considering the nonhomogeneous linear fractional differential equation with the Caputo fractional derivative

$$
\begin{aligned}
{ }_{C} D_{t_{0}, t}^{\alpha} w(t) & =k w, \quad t \geq d_{0}, \\
w\left(d_{0}\right) & =w_{0},
\end{aligned}
$$

its solution has the form [27]:

$$
w\left(t, d_{0}, w_{0}\right)=w_{0} E_{\alpha}\left(k\left(t-d_{0}\right)^{\alpha}\right) .
$$

\section{Main Results}

3.1. Stabilization. In this section, the problem of stabilization of system (1) is treated. We consider system (1) with

$$
\begin{aligned}
& A(\omega(t))=2 \omega_{1}(t) \omega_{2}(t) A_{12}+\omega_{1}^{2}(t) A_{1}+\omega_{2}^{2}(t) A_{2}, \\
& B(\omega(t))=2 \omega_{1}(t) \omega_{2}(t) B_{12}+\omega_{1}^{2}(t) B_{1}+\omega_{2}^{2}(t) B_{2} .
\end{aligned}
$$

Consider the following conditions:

(H1) $l\left(t, \omega(t), w_{1}\right)-l\left(t, \omega(t), w_{2}\right) \leq v w_{1}-w_{2}$ which implies that $l(t, \omega(t), w) \leq v w+r(t)$ where $r(t)=$ $l(t, \omega(t), 0)$.

(H2) There exist constant matrices $K_{12} \in \mathbb{R}^{p \times n}$, $K_{1} \in \mathbb{R}^{p \times n}$, and $K_{2} \in \mathbb{R}^{p \times n}$ and a symmetric positive definite matrix $P$ such that

$$
\begin{array}{r}
M\left(B_{1} K+A_{1}\right)+\left(B_{1} K+A_{1}\right)^{T} M<-d_{1} M, \\
M\left(B_{12} K+A_{12}\right)+\left(B_{12} K+A_{12}\right)^{T} M<-d_{2} M, \\
M\left(B_{2} K+A_{2}\right)+\left(B_{2} K+A_{2}\right)^{T} M<-d_{3} M,
\end{array}
$$


where $d_{1}, d_{2}$, and $d_{3}$ are strict positive reals and where $K=K_{12}+K_{1}+K_{2}$.

Theorem 1. Suppose that (H1) and (H2) hold with

$$
\left(-\frac{d}{\lambda_{\min }(M)}+2 \lambda_{\max }(M) v+\varepsilon\right)<0, \quad d=\inf \left(d_{1}, d_{2}, d_{3}\right) \text { and } \varepsilon>0,
$$

$$
g(t)=\int_{0}^{t}(t-s)^{\alpha-1} E_{\alpha, \alpha}\left(\frac{-d / \lambda_{\min }(M)+2 \lambda_{\max }(M) v+\varepsilon}{\lambda_{\max }(M)}(t-s)^{\alpha}\right) r^{2}(s) \mathrm{d} s,
$$

which is the bounded function.

Then, system (1) in closed loop with $v=K w$ is practical Mittag-Leffler stable.
Proof. Let $V(t, w)=w^{T} M w$ be a Lyapunov function candidate. Using Lemma 1, the time fractional Caputo derivative of $V$ along the trajectories of system (1) is given by

$$
\begin{gathered}
{ }^{C} D_{t_{0}, t}^{\alpha} V(t, w) \leq w^{T}\left[\omega_{1}^{2}(t)\left(M\left(A_{1}+B_{1} K\right)+\left(A_{1}+B_{1} K\right)^{T} M\right)+2 \omega_{1}(t) \omega_{2}\left(M\left(A_{12}+B_{12} K\right)+\left(A_{12}+B_{12} K\right)^{T} M\right)\right. \\
\left.+\omega_{2}^{2}(t)\left(M\left(A_{2}+B_{2} K\right)+\left(A_{2}+B_{2} K\right)^{T} M\right)\right] w+2 w^{T} M f(t, \omega(t), w),
\end{gathered}
$$

and we have

$$
\begin{aligned}
{ }^{C} D_{t_{0}, t}^{\alpha} V(t, w) & \leq\left(-\frac{d}{\lambda_{\min }(M)}+2 \lambda_{\max }(M) v+\varepsilon\right)\|w\|^{2}+\frac{\left(\lambda_{\max }(M) r(t)\right)^{2}}{\varepsilon} \\
& \leq\left(\frac{-d / \lambda_{\min }(M)+2 \lambda_{\max }(M) v+\varepsilon}{\lambda_{\max }(M)}\right) V(t, w)+\frac{\left(\lambda_{\max }(M) r(t)\right)^{2}}{\varepsilon} \\
& \leq-\rho V(t, w)+h(t),
\end{aligned}
$$

where

$$
\begin{aligned}
\rho & =\left(\frac{d / \lambda_{\min }(M)-2 \lambda_{\text {max }}(M) v-\varepsilon}{\lambda_{\text {max }}(M)}\right), \\
h(t) & =\frac{\left(\lambda_{\text {max }}(M) r(t)\right)^{2}}{\varepsilon} .
\end{aligned}
$$

Following the same proof of Theorem 1 in [3], we get the practical Mittag-Leffler stability of the closed-loop system (1).

Remark 1. The above theorem gives a fundamental result on the practical Mittag-Leffler stability of the closed-loop system (1). Note that the work introduced in [3] was among the first works which describes the concept of practical stability.

3.2. Observer Design. In this section, the problem of observer design of system (1) is treated. To obtain an estimation of the

state (we can reconstitute the state), we shall consider the following observer:

$$
\begin{aligned}
{ }_{C} D_{t_{0}, t}^{\alpha} \widehat{w}(t)= & A(\omega(t)) \widehat{w}(t)+B(\omega(t)) v(t)+f(t, \omega(t), \widehat{w}) \\
& -L(\omega(t)) C e \\
z= & C \widehat{w}(t)
\end{aligned}
$$

where $\widehat{w}(t)$ is the state estimate of $w(t), e=\widehat{w}-w$ is the error estimation, and $L(\omega(t)) \in \wp$, where

$$
L(\omega(t))=2 \omega_{1}(t) \omega_{2}(t) L_{12}+\omega_{1}^{2}(t) L_{1}+\omega_{2}^{2}(t) L_{2}
$$

The error system is defined as follows:

$$
{ }^{C} D_{t_{0}, t}^{\alpha} e(t)=(A(\omega(t))-L(\omega(t)) C) e+\Delta f
$$

where $\Delta f=f(t, \omega(t), \widehat{w})-f(t, \omega(t), w)$. 
Consider the following hypothesis:

(H3) There exist constant matrices $L_{1} \in \mathbb{R}^{n \times q}, L_{12} \in$ $\mathbb{R}^{n \times q}$, and $L_{2} \in \mathbb{R}^{n \times q}$ and a symmetric positive definite matrix $P_{1}$ such that

$$
\begin{array}{r}
M_{1}\left(-L_{1} C+A_{1}\right)+\left(-L_{1} C+A_{1}\right)^{T} M_{1}<-r_{1}^{\prime} I, \\
M_{1}\left(-L_{12} C+A_{12}\right)+\left(-L_{12} C+A_{12}\right)^{T} M_{1}<-r_{2}^{\prime} I, \\
M_{1}\left(-L_{2} C+A_{1}\right)+\left(-L_{2} C+A_{1}\right)^{T} M_{1}<-r_{3}^{\prime} I,
\end{array}
$$

where $r_{1}^{\prime}, r_{2}^{\prime}$, and $r_{3}^{\prime}$ are strict positive reals.

Theorem 2. Suppose that (H1) and (H3) hold with

$$
\left(-r^{\prime}+2 \lambda_{\max }\left(M_{1}\right) v\right)<0, \quad r^{\prime}=\inf \left(r_{1}^{\prime}, r_{2}^{\prime}, r_{3}^{\prime}\right) .
$$

Then, system (1) is Mittag-Leffler stable.

Proof. Let $W(t, w)=e^{T} M_{1} e$ be a Lyapunov function candidate. Using Lemma 1, the time fractional Caputo derivative of $V$ along the trajectories of system (20) is given by

$$
\begin{aligned}
{ }^{C} D_{t_{0}, t}^{\alpha} W(t, e) \leq & e^{T}\left[\omega_{1}^{2}(t)\left(M_{1}\left(A_{1}-L_{1} C\right)+\left(A_{1}-L_{1} C\right)^{T} M_{1}\right)+2 \omega_{1}(t) \omega_{2}(t)\left(M_{1}\left(A_{12}-L_{12} C\right)+\left(A_{12}-L_{12} C\right)^{T} M_{1}\right)\right. \\
& \left.+\omega_{2}^{2}(t)\left(M_{1}\left(A_{1}-L_{2} C\right)+\left(A_{1}-L_{2} C\right)^{T} M_{1}\right)\right] e+2 e^{T} M_{1} \Delta f .
\end{aligned}
$$

We have

$$
{ }^{C} D_{t_{0}, t}^{\alpha} W(t, e) \leq-r^{\prime} e^{2}+2 \lambda_{\max }\left(M_{1}\right) v e^{2} \leq-\widetilde{r} e^{2},
$$

where $\tilde{r}=-r^{\prime}+2 \lambda_{\max }\left(M_{1}\right) v$ which ends the proof.

\section{Numerical Examples}

4.1. Example 1. Consider the system

$$
\begin{aligned}
{ }_{C} D_{t_{0}, t}^{\alpha} x(t)= & A(\omega(t)) x(t)+B(\omega(t)) v(t) \\
& +\frac{0.1}{1+\omega_{1}^{2}(t)+\omega_{2}^{2}(t)}\left(x_{1}, \cos \left(x_{2}\right)\right), \\
y= & C x(t),
\end{aligned}
$$

where $A(\omega(t))=\omega_{1}^{2}(t) A_{1}+2 \omega_{1}(t) \omega_{2}(t) A_{12}+\omega_{2}^{2}(t) A_{2}$ and $B(\omega(t))=\omega_{1}^{2}(t) B_{1}+2 \omega_{1}(t) \omega_{2}(t) B_{12}+\omega_{2}^{2}(t) B_{2}, \quad \omega_{1}(t)=$ $\sin ^{2}(t)$, and $\omega_{2}(t)=\cos ^{2}(t)$ with $A_{1}=\left(\begin{array}{ll}-1 & 0 \\ -1 & 0\end{array}\right), A_{12}=$ $\left(\begin{array}{cc}-1 & 2 \\ 0 & -1\end{array}\right), A_{2}=\left(\begin{array}{cc}-2 & 0 \\ -2 & -3\end{array}\right), B_{1}=\left(\begin{array}{l}0.5 \\ 0.2\end{array}\right), B_{12}=\left(\begin{array}{l}0.2 \\ 0.1\end{array}\right)$, $B_{2}=\left(\begin{array}{l}0.1 \\ 0.1\end{array}\right)$, and $C=\left(\begin{array}{ll}1 & 0\end{array}\right)$.

After solving LMIs (12) using the LMI MATLAB Toolbox, we have

$$
\begin{aligned}
M & =\left(\begin{array}{cc}
0.0499 & -0.0481 \\
-0.0481 & 0.113
\end{array}\right) \\
d & =\inf \left(d_{1}, d_{2}, d_{3}\right)=\inf (0.0047,0.0058,0.0774)=0.0047
\end{aligned}
$$

Also, we have $K_{1}=\left(\begin{array}{lll}-0.7106 & 0.1393\end{array}\right), \quad K_{12}=$ $\left(\begin{array}{ll}-0.6106 & 2.5393\end{array}\right)$, and $K_{2}=\left(\begin{array}{lll}0.4106 & 0.4607\end{array}\right)$.

The constant $\varepsilon$ is chosen which is equal to 0.1 , and the Lipschitz constant $v=0.1$ satisfies the condition of Theorem 1 .

The simulation result of the present numerical example is given as follows.
Remark 2. It is clear from Figure 1 that the states of the system are practical Mittag-Leffler stable. The convergence is towards a ball of center the origin, and this validates the result of Theorem 1, that is to say the practical Mittag-Leffler stability of the system. Based on these schemes, we can say that the result obtained by Theorem 1 is satisfactory.

4.2. Example 2. Consider the system

$$
\begin{aligned}
{ }_{C} D_{t_{0}, t}^{\alpha} x(t)= & A(\omega(t)) x(t)+B(\omega(t)) v(t) \\
& +\frac{0.1}{1+\omega_{1}^{2}(t)+\omega_{2}^{2}(t)}\left(x_{1}, \cos \left(x_{2}\right)\right), \\
y= & C x(t),
\end{aligned}
$$

where $A(\omega(t))=\omega_{1}^{2}(t) A_{1}+2 \omega_{1}(t) \omega_{2}(t) A_{12}+\omega_{2}^{2}(t) A_{2}$ and $B(\omega(t))=\omega_{1}^{2}(t) B_{1}+2 \omega_{1}(t) \omega_{2}(t) B_{12}+\omega_{2}^{2}(t) B_{2}, \quad \omega_{1}(t)=$ $\sin ^{2}(t)$, and $\omega_{2}(t)=\cos ^{2}(t)$, with $A_{1}=\left(\begin{array}{cc}-1 & 0 \\ -1 & -2\end{array}\right), A_{12}=$ $\left(\begin{array}{cc}-1 & 2 \\ 0 & -1\end{array}\right), A_{2}=\left(\begin{array}{cc}-2 & 0 \\ -2 & -3\end{array}\right), B_{1}=\left(\begin{array}{l}0 \\ 0\end{array}\right), B_{12}=\left(\begin{array}{l}0 \\ 0\end{array}\right), B_{2}=$ $\left(\begin{array}{l}0 \\ 0\end{array}\right)$, and $C=\left(\begin{array}{ll}1 & 0\end{array}\right)$.

After solving LMIs (21) using the LMI MATLAB Toolbox, we have

$$
\begin{aligned}
M_{1} & =\left(\begin{array}{cc}
61.0577 & -14.2617 \\
-14.2617 & 25.4036
\end{array}\right), \\
L_{1} & =\left(\begin{array}{c}
0.3562 \\
0.8842
\end{array}\right) \\
L_{12} & =\left(\begin{array}{c}
1.5269 \\
6.787
\end{array}\right), \\
L_{2} & =\left(\begin{array}{c}
-0.2535 \\
0.6647
\end{array}\right), \\
r^{\prime} & =\inf \left(r_{1}^{\prime}, r_{2}^{\prime}, r_{3}^{\prime}\right)=\inf (50.8072,53.9269,76.2107)=50.8072 .
\end{aligned}
$$




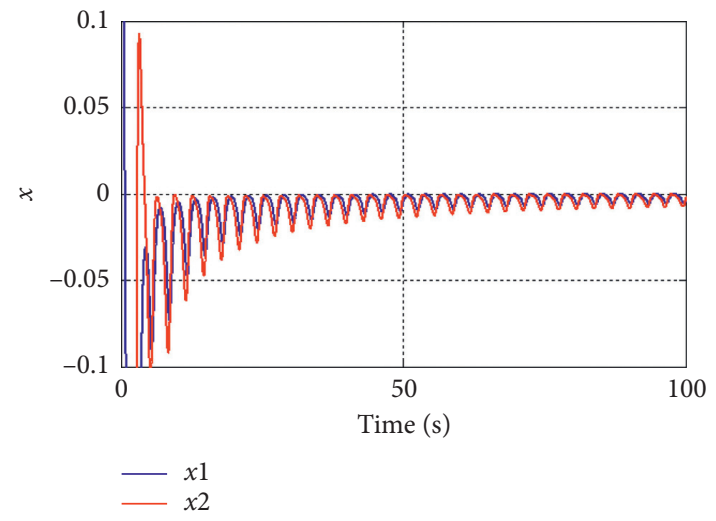

FIgURE 1: The evolution of the system states $x_{1}$ and $x_{2}$.

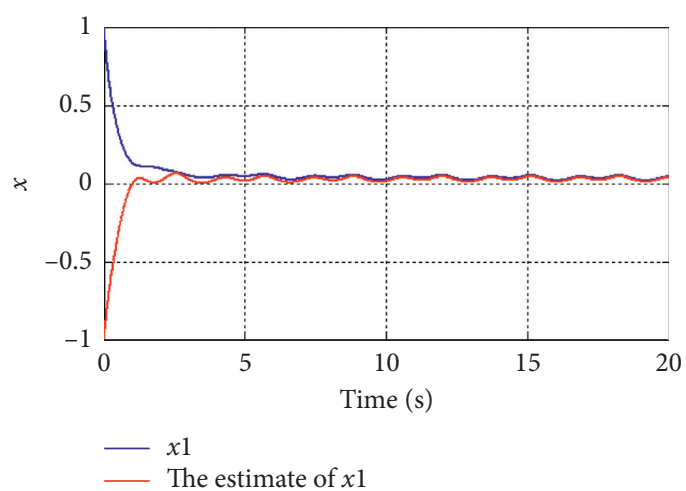

Figure 2: The evolution of the system state $x_{1}$ and its estimate $\widehat{x}_{1}$.

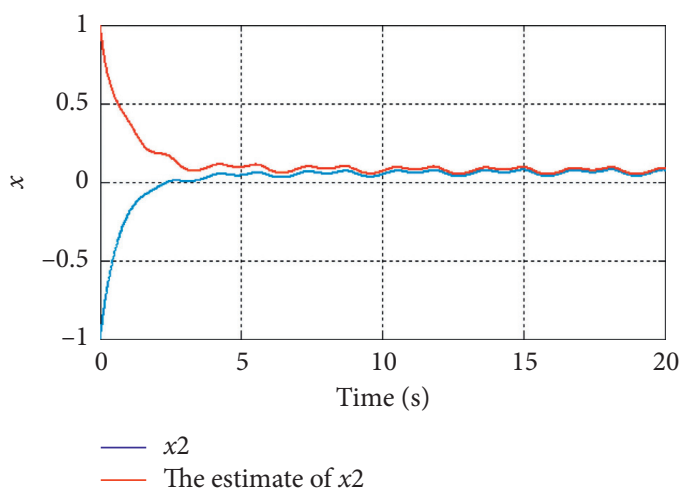

Figure 3: The evolution of the system state $x_{2}$ and its estimate $\hat{x}_{2}$.

The Lipschitz constant $v=0.3$ satisfies the condition of Theorem 2.

The simulation result of the present numerical example is given as follows.

Remark 3. It is clear from Figures 2 and 3 that the estimated states of the systems follow the actual real state which validates the observer design.

Remark 4. Figure 4 shows that the errors $e_{1}=\widehat{x}_{1}-x_{1}$ and $e_{2}=\widehat{x}_{2}-x_{2}$ converge towards the origin, and this validates

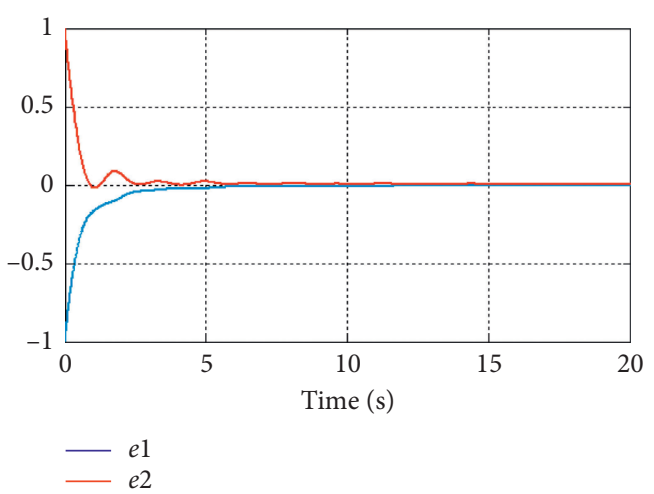

Figure 4: The evolution of the system errors $e_{1}=\widehat{x}_{1}-x_{1}$ and $e_{2}=\widehat{x}_{2}-x_{2}$.

the result of Theorem 2, that is to say the Mittag-Leffler stability of the error system. Based on these schemes, we can say that the result obtained by Theorem 2 is satisfactory.

\section{Conclusion}

In this paper, a complete methodology to solve the problem of stabilization and observer design of parameter-dependent perturbed fractional-order systems has been presented. To validate the theoretical results, a numerical example is studied in the simulation section. The simulation results show that the synchronization of the suggested chaotic fractional systems is satisfactory.

\section{Data Availability}

The present work is purely fundamental research based on theoretical analysis. It develops illustrative numerical models by means of simulations.

\section{Conflicts of Interest}

The authors declare that they have no conflicts of interest.

\section{References}

[1] A. Jmal, M. Elloumi, O. Naifar, A. Ben Makhlouf, and M. A. Hammami, "State estimation for nonlinear conformable fractional-order systems: a healthy operating case and a faulty operating case," Asian Journal of Control, vol. 22, no. 5, pp. 1870-1879, 2020.

[2] M. E. Koksal, "Time and frequency responses of non-integer order RLC circuits," AIMS Mathematics, vol. 4, no. 1, pp. 64-78, 2019.

[3] O. Naifar, A. Ben Makhlouf, M. A. Hammami, and L. Chen, "Global practical mittag leffler stabilization by output feedback for a class of nonlinear fractional-order systems," Asian Journal of Control, vol. 20, no. 1, pp. 599-607, 2017.

[4] P. Niamsup and G. Rajchakit, "New results on robust stability and stabilization of linear discrete-time stochastic systems with convex polytopic uncertainties," Journal of Applied Mathematics, vol. 2013, Article ID 368259, 10 pages, 2013.

[5] M. E. Koksal, "Stability analysis of fractional differential equations with unknown parameters," Nonlinear Analysis: Modelling and Control, vol. 24, no. 2, pp. 224-240, 2019. 
[6] A. Souahi, O. Naifar, A. B. Makhlouf, and M. A. Hammami, "Discussion on barbalat lemma extensions for conformable fractional integrals," International Journal of Control, vol. 92, no. 2, pp. 234-241, 2019.

[7] O. Naifar, A. Jmal, A. M. Nagy, and A. B. Makhlouf, "Improved quasiuniform stability for fractional order neural nets with mixed delay," Mathematical Problems in Engineering, vol. 2020, Article ID 8811226, 7 pages, 2020.

[8] G. Rajchakit, P. Chanthorn, M. Niezabitowski, R. Raja, D. Baleanu, and A. Pratap, "Impulsive effects on stability and passivity analysis of memristor-based fractional-order competitive neural networks," Neurocomputing, vol. 417, pp. 290-301, 2020.

[9] M. Rajchakit and G. Rajchakit, "Mean square robust stability of stochastic switched discrete-time systems with convex polytopic uncertainties," Journal of Inequalities and Applications, vol. 135, no. 1, 2012.

[10] S. Boyd, L. EL Ghaoui, E. Feron, and V. Balakrishnan, Linear Matrix Inequalities in System and Control Theory, SIAM, Philadelphia, PA, USA, 1994.

[11] P. Gahinet, A. Nemirovski, A. J. Laub, and M. Chilali, "The LMI control toolbox," in Proceedings of 1994 33rd IEEE Conference on Decision and Control, pp. 2038-2041, Lake Buena Vista, FL, USA, December 1994.

[12] H. Gao, P. Shi, and J. Wang, "Parameter-dependent robust stability of uncertain time-delay systems," Journal of Computational and Applied Mathematics, vol. 206, no. 1, pp. 366-373, 2007.

[13] U. Jonsson and A. Rantzer, "System with uncertain parameters-time-variations with bounded derivatives," in Proceedings of 1994 33rd IEEE Conference on Decision and Control, pp. 3074-3079, Lake Buena Vista, FL, USA, December 1994.

[14] A. B. Abdallah, M. Dlala, and M. A. Hammami, "A new Lyapunov function for stability of time-varying nonlinear perturbed systems," Systems \& Control Letters, vol. 56, no. 3, pp. 179-187, 2007.

[15] S. Dasgupta, G. Chockalingam, B. D. O. Anderson, and M. Minyue Fe, "Lyapunov functions for uncertain systems with applications to the stability of time varying systems," IEEE Transactions on Circuits and Systems I: Fundamental Theory and Applications, vol. 41, no. 2, pp. 93-106, 1994.

[16] B. R. Barmish, "Necessary and sufficient conditions for quadratic stabilizability of an uncertain system," Journal of Optimization Theory and Applications, vol. 46, no. 4, pp. 399-408, 1985.

[17] M. A. Hammami, "Global convergence of a control system by means of an observer," Journal of Optimization Theory and Applications, vol. 108, no. 2, pp. 377-388, 2001.

[18] Y.-J. Sun, "Global stabilizability of uncertain systems with time-varying delays via dynamic observer-based output feedback," Linear Algebra and Its Applications, vol. 353, no. 13, pp. 91-105, 2002.

[19] A. B. Makhlouf, M. A. Hammami, and K. Sioud, "Stability of fractional order nonlinear systems depending on a parameter," Bulletin of the Korean Mathematical Society, vol. 54, no. 4, pp. 1309-1321, 2017.

[20] G.-H. Yang and J. Dong, "Robust stability of polytopic systems via affine parameter-dependent Lyapunov functions," in Proceedings of the 48th IEEE Conference on Decision and Control (CDC), Shanghai, China, December 2009.

[21] F. Chen, S. Kang, and F. Li, "Stability and stabilization for polytopic LPV systems with parameter-varying time delays," Mathematical Problems in Engineering, vol. 2019, Article ID 4924963, 12 pages, 2019.
[22] L. Chen, R. Wu, Y. He, and L. Yin, "Robust stability and stabilization of fractional-order linear systems with polytopic uncertainties," Applied Mathematics and Computation, vol. 257, pp. 274-284, 2015.

[23] C. Lin, Q.-G. Wang, and T. H. Lee, "A less conservative robust stability test for linear uncertain time-delay systems," IEEE Transactions on Automatic Control, vol. 51, no. 1, pp. 87-91, 2006.

[24] D. C. W. Ramos and P. L. D. Peres, "An LMI condition for the robust stability of uncertain continuous-time linear systems," IEEE Transactions on Automatic Control, vol. 47, no. 4, pp. 675-678, 2002.

[25] K. Diethelm, The Analysis Of Fractional Differential Equations, An Application Ori- Ented, Exposition Using Differential Operators of Caputo Type, Springer-Verlag, Heidelbereg, Germany, 2010.

[26] A. A. Alikhanov, "A priori estimates for solutions of boundary value problems for fractional-order equations," Differential Equations, vol. 46, no. 5, pp. 660-666, 2010.

[27] A. A. Kilbas, H. M. Srivastava, and J. J. Trujillo, Theory and Application of Fractional Differential Equations, Elsevier, New York, NY, USA, 2006. 